\title{
A Mouse Model of Lethal Synergism Between Influenza Virus and Haemophilus influenzae
}

\author{
Lian Ni Lee, ${ }^{*}$ Peter Dias, ${ }^{*}$ Dongun Han, ${ }^{*}$ \\ Sorah Yoon, ${ }^{*}$ Ashley Shea, ${ }^{*}$ Vladislav Zakharov, ${ }^{\dagger}$ \\ David Parham, ${ }^{\dagger}$ and Sally R. Sarawar ${ }^{*}$ \\ From Viral Immunology,* Torrey Pines Institute for Molecular \\ Studies, San Diego, California; and the Department of \\ Pathology, ${ }^{\dagger}$ University of Oklahoma Health Sciences Center, \\ Oklahoma City, Oklaboma
}

Secondary bacterial infections that follow infection with influenza virus result in considerable morbidity and mortality in young children, the elderly, and immunocompromised individuals and may also significantly increase mortality in normal healthy adults during influenza pandemics. We herein describe a mouse model for investigating the interaction between influenza virus and the bacterium Haemophilus influenzae. Sequential infection with sublethal doses of influenza and $H$. influenzae resulted in synergy between the two pathogens and caused mortality in immunocompetent adult wild-type mice. Lethality was dependent on the interval between administration of the bacteria and virus, and bacterial growth was prolonged in the lungs of dual-infected mice, although influenza virus titers were unaffected. Dual infection induced severe damage to the airway epithelium and confluent pneumonia, similar to that observed in victims of the 1918 global influenza pandemic. Increased bronchial epithelial cell death was observed as early as 1 day after bacterial inoculation in the dual-infected mice. Studies using knockout mice indicated that lethality occurs via a mechanism that is not dependent on Fas, CCR2, CXCR3, interleukin-6, tumor necrosis factor, or Toll-like receptor-4 and does not require $\mathbf{T}$ or $B$ cells. This model suggests that infection with virulent strains of influenza may predispose even immunocompetent individuals to severe illness on secondary infection with $H$. influenzae by a mechanism that involves innate immunity, but does not require tumor necrosis factor, interleukin-6, or signaling via Toll-like receptor-4. (Am J Pathol 2010, 176:800-811; DOI: 10.2353/ajpath.2010.090596)
Infections with influenza virus cause mild to severe respiratory illness and may result in death in vulnerable human populations. ${ }^{1-4}$ On average, influenza causes three to five million cases of severe illness per year worldwide and over 200,000 hospitalizations and 36,000 deaths in the United States alone. ${ }^{1} 5$ to $20 \%$ of the US population are infected annually. While healthy adults typically experience only acute uncomplicated infection, influenza virus predisposes the lungs to bacterial co-infections, ${ }^{5-7}$ which cause significant additional morbidity, particularly in young children, elderly and immunocompromised individuals. ${ }^{8-12}$ Secondary bacterial infections may also significantly increase mortality in the population as a whole during influenza pandemics. ${ }^{13-16}$ For example, in the 1918 influenza pandemic, which killed approximately 50 million people worldwide, while infection with the virus alone could be lethal, the majority of deaths appeared to result from secondary bacterial pneumonia. ${ }^{16-19}$ The most common bacterial agents mediating such secondary infections in the U.S. are Streptococcus pneumoniae, Staphylococcus aureus, and Haemophilus influenzae. ${ }^{20-23}$

$H$. influenzae is a small Gram-negative coccobacillus that exists in capsulated or non-capsulated forms. $H$. influenzae is a common cause of otitis media, acute sinusitis, bronchitis, pneumonia and exacerbations of chronic obstructive pulmonary disease. ${ }^{24-29}$ A vaccine against $H$. influenzae type b (Hib) has greatly reduced the incidence of invasive disease, such as meningitis, caused by this organism in children under 5 years of age ${ }^{30-33}$ However, Hib invasive disease in children remains a problem in countries where the vaccine is not

Supported by the National Institutes of Health Grant Al59429 and by a grant from the Infectious Disease Science Center.

L.N.L. and P.D. contributed equally to this study.

Accepted for publication October 27, 2009.

Current address of L.L.: Nuffield Department of Medicine, Oxford University, Oxford, United Kingdom; current address of D.H.: Animal Care Division, Cheonan Yonam College, Republic of Korea; current address of S.Y.: Virology Division, National Veterinary Research and Quarantine Service, Ministry for Food, Agriculture, Forestry and Fisheries, Republic of Korea.

Address reprint requests to Sally R. Sarawar, Ph.D., Viral Immunology, Torrey Pines Institute for Molecular Studies, 3550 General Atomics Court, San Diego, CA 92121. E-mail: ssarawar@tpims.org 
widely available. ${ }^{32,34,35}$ Furthermore, other encapsulated and non-typable (NTHi) forms are increasing in frequency as causes of illness in young children. ${ }^{28,29,36}$ During the 1918 influenza pandemic, $H$. influenzae was often isolated from the autopsied lungs of young adults, a subpopulation who do not usually die from influenza infection. ${ }^{16}$

Early studies by Shope ${ }^{37}$ showed that infection of pigs with both influenza virus and $H$. influenzae suis resulted in severe disease or death, whereas the individual agents induced only mild infection. Similarly, Orticoni et $\mathrm{al}^{38}$ reported that administration of both filtrates of nasal secretions from 1918 influenza patients and $H$. influenzae caused a lethal disease in guinea pigs, but there was no effect if either agent was administered alone. Influenza also increases the susceptibility of new-born rats to $H$. influenzae-induced meningitis ${ }^{39}$ and synergizes with the bacteria in the development of otitis media in the chinchilla. ${ }^{40} \mathrm{~A}$ single study conducted in 1945 showed that infection with both influenza virus and $H$. influenzae killed mice at doses that were sublethal when either agent was administered alone. ${ }^{41}$ However, this study pre-dated modern immunological techniques, precluding assessment of the underlying mechanism.

To investigate the pathobiological mechanisms further, we established a model of influenza and $H$. influenzae co-infection in mice. Herein, we report that $H$. influenzae synergizes with influenza virus to cause more severe disease in immunocompetent adult mice, leading to $100 \%$ lethality at doses that cause no mortality when the agents are give individually. The mechanism leading to disease exacerbation does not involve T or B cells, and thus appears to be mediated by innate immunity. However, tumor necrosis factor (TNF), interleukin-6 (IL-6), and Toll-like receptor (TLR)-4 are not essential for synergistic lethality in this model.

\section{Materials and Methods}

\section{Preparation of Virus Stock}

Influenza A/Puerto Rico/8/34 (A/PR/8/34; flu) was obtained from the American Type Culture Collection (ATCC; VR-1469, Manassas, VA), and stocks were grown in Madin Darby canine kidney (MDCK) cells (ATCC). Infectious virus titers were determined by plaque formation in monolayers of MDCK cells by the method of Tannock et al. ${ }^{42}$

\section{Preparation of Bacterial Stock}

The kanamycin-resistant encapsulated type b strain (Eagan) H. influenzae H338 (Hib) was a kind gift from Drs. J.M. Wilson and J. Weiser. ${ }^{43}$ This kanamycin-resistant strain was used to exclude possible contamination by other bacteria present in mouse lungs or introduced during preparation of bacterial stocks. Stocks were grown in brain heart infusion broth (BD Biosciences, Sparks Glencoe, MD) supplemented with $2 \%(\mathrm{v} / \mathrm{v})$ Fildes Enrichment (BD Biosciences), $2 \mu \mathrm{g} / \mathrm{ml}$ NAD (Sigma-Aldrich, Milwaukee, WI) and $20 \mu \mathrm{g} / \mathrm{ml}$ kanamycin sulfate (Sigma-Aldrich) to mid-log phase, recovered by centrifugation, resuspended in growth media containing $16 \%(\mathrm{v} / \mathrm{v})$ glycerol, aliquoted and stored at $-80^{\circ} \mathrm{C}$ until use. The stocks were quantified by plating $10 \mu \mathrm{l}$ serial dilutions in sterile phosphate buffered saline (PBS) (Mediatech, Herndon, VA) onto chocolate agar plates containing $20 \mu \mathrm{g} / \mathrm{ml}$ kanamycin sulfate. Plates were incubated for 24-48 hours and the characteristic buff gray colonies were counted.

\section{Mouse Strains and Infection}

C57BL/6, C57BL/10ScSnJ, C57BL/10ScNJ (TLR4-/-), RAG-/-, IL6-/-, B6Fas $-/-$, and TNFR1R2-/- mice between 8 and 12 weeks of age were obtained from Jackson Laboratory (Bar Harbor, ME). CXCR3-/- mice were obtained from Dr. Craig Gerard (Children's Hospital and Harvard Medical School, Boston, Massachusetts) and CCR2-/- mice were from Dr. Israel Charo (Gladstone Institute of Cardiovascular Disease, San Francisco, $\mathrm{CA})$. Mice were bred and housed under specific pathogen-free (SPF) conditions in the animal resource center at the Torrey Pines Institute for Molecular Studies. All experiments were performed in accordance with a protocol approved by the Institutional Animal Care and Use Committee of Torrey Pines Institute for Molecular Studies, in compliance with the National Institutes of Health U.S. Public Health Service guidelines for the care and use of animals. Mice were anesthetized with Avertin (2,2,2-tribromoethanol) and infected intranasally (i.n.) with 30- $\mu$ l volume of between 1 and 1000 plaque-forming units (PFU) of influenza in PBS. At various time points after primary influenza infection, some groups of mice were anesthetized again and infected i.n. with $30 \mu \mathrm{l}$ of $1-10^{7}$ colony-forming units (CFU) of Hib bacteria in PBS or given PBS only as control. Some mice were only infected with Hib. Survival was monitored daily after the second infection.

\section{Bronchoalveolar Lavage (BAL) and Lung Homogenate Preparation}

At various times after infection, the mice were sacrificed by terminal anesthesia with Avertin. Bronchoalveolar lavage was performed via cannulation of the trachea. The lungs were lavaged three times with $1 \mathrm{ml}$ of ice-cold PBS; lungs were then harvested and a 10\% (w/v) homogenate was prepared in PBS using a Tissue Tearor homogenizer (Fisher Scientific, Pittsburgh, PA). Bacteria were quantified by plating $10-\mu l$ serial dilutions of the BAL onto chocolate agar plates containing kanamycin, as described above. Levels of flu virus in the lung homogenates were quantitated by plaque assay on MDCK monolayers as described above.

\section{Lung and Brain Histopathology}

Mice were infected with flu alone (day 0), Hib alone (day 3 ), or both agents 3 days apart. At day 9 , the mice were sacrificed and the lungs and brains were removed and fixed in 10\% neutral buffered formalin (Sigma Aldrich, 
A

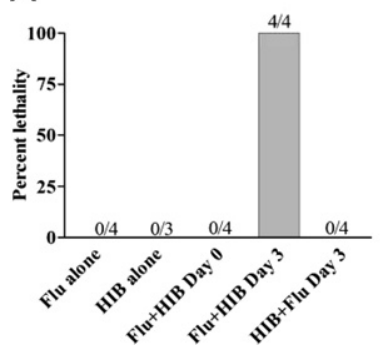

B

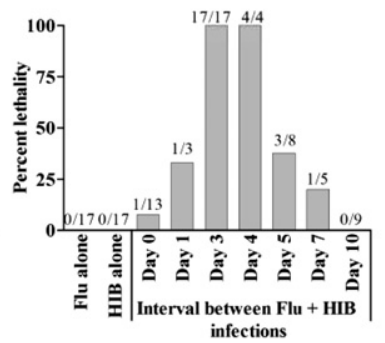

C

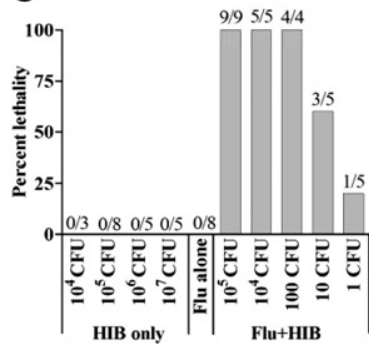

D

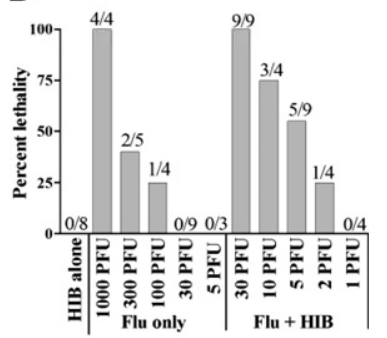

Figure 1. Lethal synergy between influenza virus and Hib. A: Lethal synergy between influenza virus and Hib occurs after sequential but not after simultaneous infection. Groups of four female C57BL/6 mice were infected intranasally with 30 PFU of influenza A/PR8/34 (Flu) in PBS, mock-infected with PBS or given a combination of 30 PFU flu and $10^{5}$ CFU Hib in PBS. 3 days later, mice were infected with $10^{5}$ CFU Hib in PBS or mock-infected with PBS. An additional group of mice was infected first with Hib and then flu 3 days later. B: Lethality is dependent on the time interval between exposure to flu and Hib. The two agents were either administered at the same time (day 0) or Hib was given 3, 4, 5, 7 or 10 days after flu. C: Effect of altering the dose of flu on lethality. Mice were infected with $10^{5} \mathrm{CFU}$ Hib, varying doses of flu or both agents 3 days apart. D: Effect of altering the dose of Hib on lethality. Mice were infected i.n. with 30 PFU flu, varying doses of Hib or both agents 3 days apart. Mice were observed for 25 days after infection. Data in each chart are shown as percent lethality. The number of mice dead/number of initial mice in each group is shown above the bar.

Milwaukee, WN). Tissues were embedded in paraffin wax, sectioned and hematoxylin and eosin stained sections were prepared and examined by microscopy to determine the extent of inflammation. Three parameters were evaluated in the lungs: alveolitis, bronchiolitis, and follicular bronchitis. Alveolitis was defined as areas of alveolar septal thickening and pulmonary fibrosis. Bronchiolitis was defined as polymorphonuclear and mononuclear cell infiltrates that condensed into prominent peribronchiolar polymorphonuclear cell accumulations. Follicular bronchitis was defined as mononuclear cell infiltrates that condensed into prominent peribronchial lymphoid accumulations. Bronchiolitis, follicular bronchitis, and alveolitis were blindly scored on a scale of 0 to 3 depending on the degree of inflammatory cell infiltration and the percentage of the tissue affected: 0, normal (very few or no inflammatory cells); 1 , mild (<20\%); 2 , moderate (20 to $40 \%)$; 3 , severe $(>40 \%)$.

\section{Flow Cytometric Analysis}

BAL cells were stained with phycoerythrin or fluorescein isothiocyanate-conjugated antibodies, as previously described. ${ }^{44}$ The resulting populations were analyzed by flow cytometry using a Becton Dickinson FacsCalibur (San Jose, CA).

\section{Cytokine Enzyme-Linked Immunosorbent Assay (ELISA)}

ELISAs for MCP-1, IL-6, TNF, and IP-10 were performed on BAL fluid from infected mice using kits from R\&D Systems (Minneapolis, MN) according to the manufacturer's instructions. The detection limit of these assays was between 8 and $16 \mathrm{pg} / \mathrm{ml}$.

\section{Results}

\section{Sequential Infection of Mice with Sublethal Doses of Influenza and Hib Results in Death}

Single infections with influenza and Hib in mice have been described extensively. Intranasal infection with a
Iow (30 PFU) dose of influenza A/PR8/34 or with $10^{5} \mathrm{CFU}$ of Hib alone was not lethal in adult wild type C57BL/6 mice (Figure 1A). By contrast, when mice were infected i.n. with 30 PFU influenza and 3 days later with $10^{5} \mathrm{CFU}$ $\mathrm{Hib}$, the co-infected mice typically died between 9 and 14 days postinfluenza infection. Control mice that were infected with influenza and then mock-infected with PBS, or mock infected with PBS on both occasions, survived and remained healthy throughout the 25-day observation period. Influenza and Hib therefore appear to synergize, resulting in more severe disease in this mouse model. Further studies to define the model showed that this synergistic effect only occurred when the agents were administered sequentially. Almost all mice survived coinfection when $30 \mathrm{PFU}$ influenza virus and $10^{5} \mathrm{CFU}$ of Hib were administered at the same time (Flu + Hib day 0). Furthermore, no lethality occurred when the order of sequential infection was reversed, i.n. infection with Hib followed by infection with influenza 3 days later (Figure 1A). These mice appeared healthy throughout the 25-day observation period. Similar results were obtained with kanamycin resistant (Figure 1A) and non-kanamycin resistant (data not shown) variants of the Eagan strain of Hib.

As the synergistic effect was observed only during sequential infection, experiments were conducted to determine whether the interval between influenza and Hib infections affected the outcome. Groups of mice were first infected i.n. with 30 PFU of influenza, and then with Hib, either simultaneously or up to 7 days post-influenza infection (Figure 1B). Low percentage mortality was observed when the interval was increased to 2 days. However, $100 \%$ lethality resulted when the Flu and Hib were given 3-4 days apart. The percentage mortality was markedly reduced when the interval was lengthened to 5-7 days and no mortality was observed in mice that were infected with the two agents 10 days apart. Therefore, the time interval between infections is critical for lethal synergy.

The effect of the dose of influenza was also examined. As shown in Figure 1C, a dose of 1000 PFU of influenza resulted in 100\% mortality in singly-infected mice, while some mortality occurred at a dose of 100 PFU. In con- 

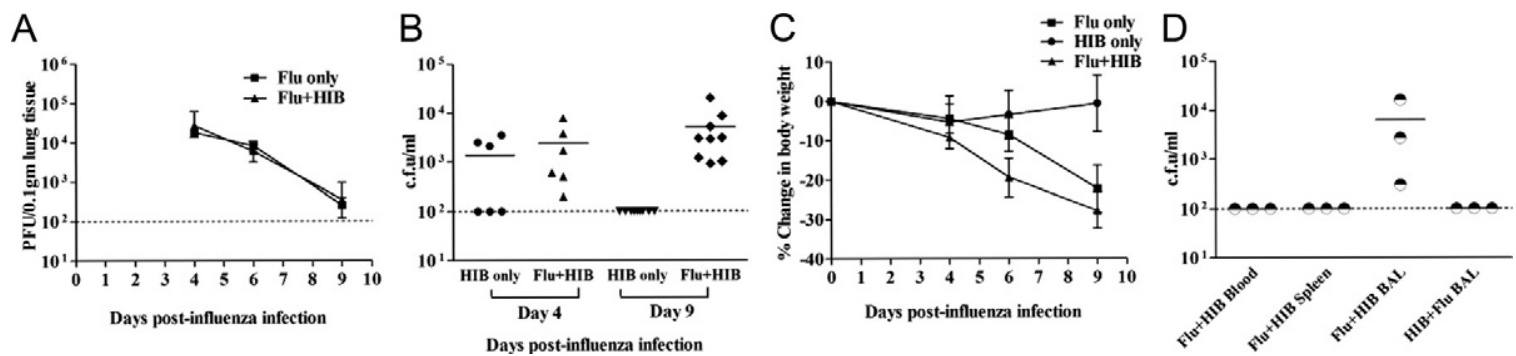

Figure 2. Sequential infection with influenza virus and Hib has no effect on viral titers but prolongs bacterial growth. A: Titers of influenza virus in the lungs. Two groups of C57BL/6 mice were infected i.n. with 30 PFU flu at day 0. At day 3, one group was also infected with $10^{5}$ CFU Hib (double-infected). Lungs were collected at day 4,6 , and 9 post-flu infection from flu only (Flu) or double-infected groups. Infectious virus titers in $10 \%$ lung homogenates were determined by plaque assay on MDCK cells. Data shown are the mean titers of three mice at each time point, with the error bars depicting the SD. The experiment was performed twice. B: Bacterial counts in BAL fluid. One group of C57BL/6 mice was infected i.n. with 30 PFU flu at day 0 and then infected i.n. with $10^{5}$ CFU of Hib at day 3 (Flu + Hib), together with another group of mice that were not previously infected with flu (Hib only). BAL fluid (total volume 1 ml) was obtained from the lungs at days 4 and 9 post-flu infection. Bacterial counts in BAL fluid were determined by titration on chocolate agar. Data shown are CFU bacteria/ml BAL fluid from two separate experiments. C: Weights of mice. Mice in each of the three groups were weighed at the specified time points. D: Comparison of bacterial counts in the spleen, blood and BAL of influenza and Hib co-infected mice at day 9 after infection with influenza. Mice were infected as above (Flu-Hib) or with $10^{5}$ CFU of HIB followed 3 days later by 30 PFU flu (Hib-Flu). BAL was collected as described above, spleens were homogenized in 1 ml of PBS and blood was collected in heparinized tubes. Bacterial counts were determined as described above.

trast, doses of influenza that were 30 PFU or fewer did not by themselves kill the infected host. However, when combined with $10^{5} \mathrm{CFU} \mathrm{Hib}, 55 \%$ lethality was observed in mice that were infected with only 5 PFU of influenza, suggesting that even low dose influenza infection in adult wild-type mice may increase the predisposition to complications arising from a secondary bacterial infection.

The effect of the dose of Hib on the survival of single and dual-infected mice was examined by infecting mice with a range of concentrations of Hib and monitoring the infected animals for 25 days. The results in Figure 1D demonstrate that adult wild-type C57BL/6 mice are able to tolerate i.n. infection of up to $10^{7} \mathrm{CFU}$ of Hib without any apparent ill effects. By contrast, when combined with a prior influenza infection, 100\% lethality was observed after administration of as little as $10 \mathrm{CFU}$ of bacteria, suggesting that influenza infection dramatically increases the susceptibility of the host to secondary bacterial infection.

\section{Dual-Infected Mice Are Able to Control Influenza Virus Infection but Show Compromised Clearance of Hib from the Lungs}

We assessed levels of influenza virus in the lungs of mice infected with only influenza or both agents to determine whether lethality was the result of uncontrolled viral spread. As shown in Figure 2, A and C, the change in body weight or viral titer in the lungs of mice infected with influenza and Hib did not differ from that of mice infected with influenza alone at days 4 or 6 postinfection. Furthermore, the level of virus was reduced to the same extent in both groups at day 9, indicating influenza virus replication in the lung was controlled equally well in influenza and influenza-Hib infected mice.

It is possible that a prior infection with influenza virus could lead to increased growth or persistence of bacteria in the lungs, which may contribute to host mortality. The bacterial counts of BAL fluid from the lungs of single and double-infected mice support this hypothesis. As shown in Figure 2B, mice that were infected with Hib only did not have detectable levels of bacteria in BAL 6 days after infection (this corresponds to day 9 post-influenza infection) while mice that were previously infected with influenza virus still had significant numbers of bacteria in the lungs at this time point. No bacteria were detected in the blood or spleen (Figure 2D). In addition, no bacteria were detected in the lungs of mice that were infected with Hib and influenza in the reverse order (Hib 3 days before influenza virus, Figure 2D).

\section{Severe Lung Damage after Sequential Infection with Influenza and $\mathrm{H}$. influenzae}

To investigate the inflammatory response and type of tissue damage preceding death, groups of mice were infected with influenza virus or Hib alone or sequentially with influenza virus and Hib as before. As co-infected mice usually died between 9-14 days after influenza infection, the co-infected mice were sacrificed at days 4 and 9 postinfluenza infection. Mice infected with influenza virus or Hib only were also killed at the same time. The lungs were removed, fixed in formalin, embedded, and hematoxylin and eosin-stained sections were prepared. Representative sections are shown in Figure 3.

Pulmonary histopathological changes were observed 4 and 9 days following infection with Hib alone, influenza virus alone, and Hib with influenza virus. Lungs from control mice showed no significant inflammation (Figure $3 A)$. Lungs from the Hib group acquired a mild, patchy mixed lymphocytic/neutrophilic bronchitis after day 4, while lymphocytes accumulated within and around bronchi 4 days after influenza virus inoculation, with progressive infiltration into alveolar septa and production of patchy interstitial pneumonitis at 9 days (Figure 3, A and B). At day 9, there was also progressive destruction of bronchial epithelium, accompanied by evidence of epithelial regeneration (Figure 3A).

In the Hib plus influenza virus group, the most characteristic lesion was a suppurative bronchitis and bronchiolitis characterized at day 4 postinoculation by prominent intrabronchial neutrophilic infiltrates and epithelial necro- 
A Uninfected

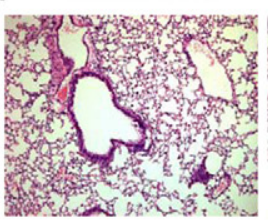

Hib

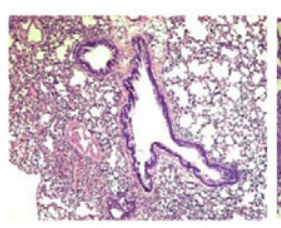

Flu

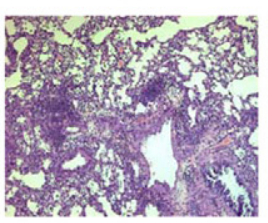

$\mathrm{Flu}+\mathrm{Hib}$

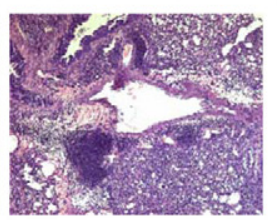

B Hib

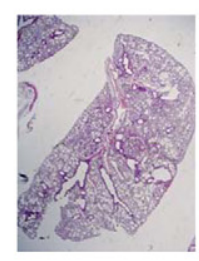

Flu

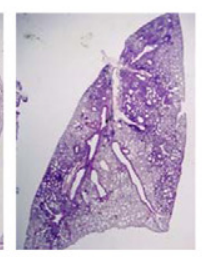

Flu + Hib

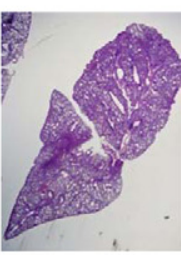

C

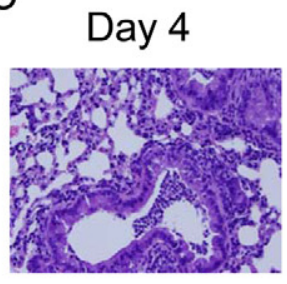

Day 4

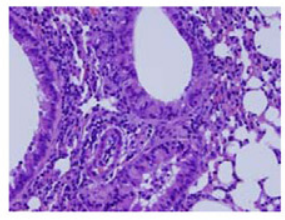

Day 9

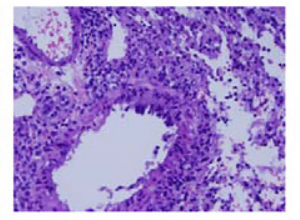

D

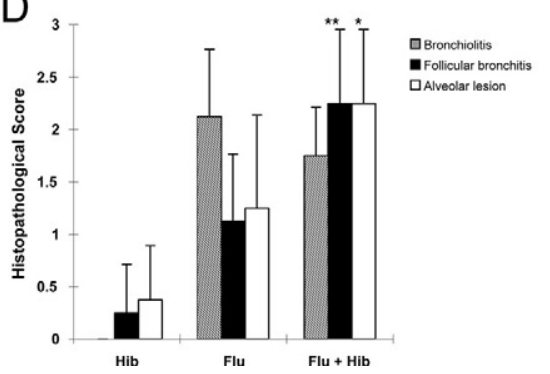

Figure 3. Lung histopathology demonstrates that mice co-infected with influenza virus and Hib show more inflammation and epithelial cell destruction than those infected by either agent alone. Groups of four mice were infected intranasally with 30 PFU influenza A/PR8 (flu), $10^{5}$ CFU H. influenzae (Hib) or both agents 3 days apart (Flu + Hib). Lung samples were collected 4 and 9 days after influenza infection (1 and 6 days after Hib infection). Lung samples from uninfected mice were also collected as controls. Lungs were embedded, sectioned and hematoxylin and eosin (H\&E)-stained sections were prepared and examined by microscopy. Similar results were obtained in two independent experiments. Photomicrographs of representative H\&E sections are shown. A: Comparative histology of lungs from control (uninfected), Hib, Flu, or Flu + Hib-infected mice. Tissues were harvested 9 days after influenza infection (6 days after Hib infection). Lungs from mice infected with Hib alone show low levels of inflammation, while those of mice infected with flu alone contain intra-alveolar infiltrates of lymphocytes comprising a peribronchial interstitial pneumonitis with retention of alveolar spaces. In contrast, in mice infected sequentially with both agents, a pneumonitis obliterates the alveolar spaces, which are filled with neutrophils and fluid. Lymphocytes infiltrate the pulmonary interstices and extensive denudation of the bronchial epithelium is apparent. Original magnification, $\times 40$. B: Low power comparison of lung tissue from Hib, Flu or Flu + Hib infected mice prepared as described in $\mathbf{A}$. There is a progressive increase in inflammation as assessed by the degree of cellularity. Note the bronchiolar pattern in the viral pneumonia, and the focal confluent bronchopneumonia in lungs from mice infected with both agents. In comparison, a lung from a mouse infected with Hib alone shows recovery of a normal bronchial pattern and little obvious pneumonia. Original magnification, $\times 10$. C: Histopathological features of lungs from co-infected mice at days 4 and 9 after infection with influenza (days 1 and 6 after Hib). Left: Day 4. Acute necrotizing bronchitis with neutrophils. Center: Day 4. Bronchial necrosis with apoptotic bodies and peribronchial inflammation. Right: Day 9. Erosive bronchiolitis. Original magnification, $\times 100$. D: Histopathological scores for H\&E sections of lungs from mice infected with Hib, Flu or Flu + Hib prepared as in A. Tissues were harvested 9 days after influenza infection (6 days after Hib infection). Bronchiolitis, follicular bronchitis and alveolitis were blindly scored on a scale of 0 to $3: 0$, normal; 1 , mild; 2 , moderate; 3 , severe. There was a significant difference in the scores for follicular bronchitis and alveolitis between groups of mice infected with Flu alone and Flu + Hib. ${ }^{*} P<0.05$. ${ }^{* *} P<0.01$ (Mann-Whitney rank sum test). Scores for lungs from mice infected with Hib alone were significantly lower in all categories than those for mice infected with both flu $\&$ Hib $(P<0.001$ for all categories). Data are combined from two independent experiments, with four mice per group in each experiment.

sis (Figure 3C). This bronchiolitis was most prominent in the Hib plus influenza group, although foci with similar changes were noted in the influenza group at day 9 (Figure 3, A and B). However, with combined infection, the bronchial epithelium showed severe denudation with no evidence of recovery, confluent alveolar pneumonia with suppurative exudates, and epithelial cell apoptosis in the airways (Figure $3, \mathrm{~A}$ and $\mathrm{C}$ ).

Histopathology was also was evaluated quantitatively using three parameters: alveolitis, bronchiolitis, and follicular bronchitis, as described in Materials and Methods. Mice infected with influenza virus followed by Hib had significantly increased pathological lesion scores of the lungs, as compared with Hib or influenza only infected mice (Figure 3D). However, similar scores for bronchiolitis were noted in the dual infected and influenza virus group, while follicular bronchitis and alveolitis were significantly increased in influenza virus with Hib-infected group compared with the singly infected groups (Figure 3D).

Although co-infection with influenza virus and Hib is associated with meningitis in young children, sections of the brains of mice co-infected in our model appeared normal with no evidence of meningitis (Figure 4, A and B). Furthermore, we found no evidence of bacteremia or spread of bacteria to organs such as the spleen (Figure
2D). Overall, our observations are consistent with the idea that lethality is caused by damage to the lungs.

\section{The Adaptive Immune Response is Not Essential for the Development of Lethal Synergy}

Mice that were sequentially infected with influenza virus and Hib typically died between 9 and 14 days after

A
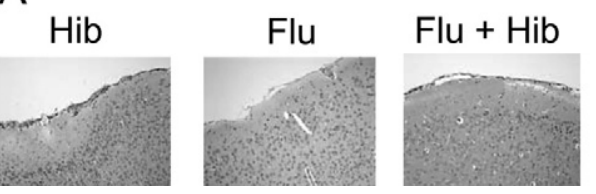

B

Figure 4. Brain histopathology of mice infected with influenza virus and Hib. Brain tissues were also collected at day 9 postinfection with influenza from the groups of infected mice described in Figure 3. Tissues were fixed, embedded in paraffin wax, sectioned and H\&E-stained sections were prepared and examined by microscopy. Photomicrographs of representative H\&E sections are shown. A: H\&E sections of brain tissue from Hib, Flu or Flu + Hib-infected mice: Cerebral cortex with intact laminar architecture and no evidence of pia-arachnoidal inflammation. Objective magnification, $\times 20$. B: H\&E section of brain tissue (Brain) from Hib + Flu-infected mouse. Cerebellar cortex with intact molecular, Purkinje cell, and granular cell layers and no evidence of pia-arachnoidal inflammation. Objective magnification, $\times 20$. 


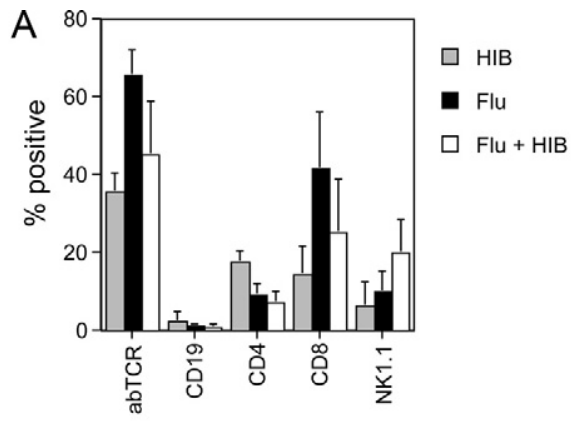

B

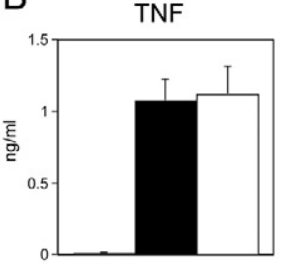

MCP-1
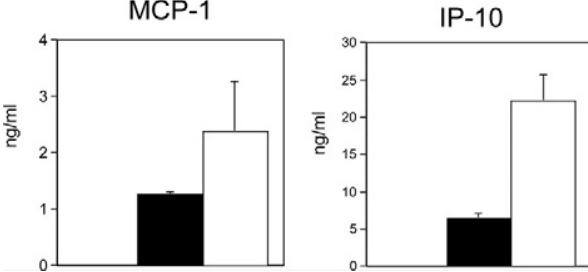

Figure 5. Cell types and cytokines in bronchoalveolar lavage from mice infected with influenza virus and Hib. Groups of three or four mice were infected intranasally with 30 PFU influenza A/PR8 (flu), $10^{5}$ CFU H. influenzae (Hib) or both agents 3 days apart (Flu + Hib). Bronchoalveolar lavage samples were collected 9 days after influenza infection 6 days after Hib infection) and centrifuged to produce a cellular fraction for flow cytometric analysis of lymphocyte subsets and a cell-free supernatant for evaluation of cytokine concentrations by ELISA. A: Flow cytometric analysis of BAL cells. Cells were stained with phycoerythrin or fluorescein isothiocyanate-conjugated antibodies to the $\alpha \beta$ T cell receptor (abTCR), CD4 or CD 4 (T cells), CD19 (B cells) or NK1.1 (NK cells and NK T cells). The resulting populations were analyzed by flow cytometry using a lymphocyte gate. Mean percentage positive cells + SD are shown. B: Cytokine and chemokine concentrations in BAL. Concentrations of IL-6, TNF, MCP-1 and IP-10 were determined by ELISA. Data represent mean concentrations + SEM for two independent experiments (with the exception of MCP-1, which was from a single experiment). IL-6, TNF, IP-10, and MCP-1 were present at low to undetectable levels in BAL from mice infected with Hib alone. Levels of IP-10 were significantly increased in the BAL of coinfected mice compared with that of mice infected with flu only ( $P=0.001$, Mann-Whitney rank sum test)

influenza infection. This timeframe coincides with the development of $\mathrm{T}$ cell responses. Figure $5 \mathrm{~A}$ shows that $\mathrm{T}$ cells comprised a significant proportion of the BAL population at day 9, while very few $\mathrm{CD}_{19}{ }^{+} \mathrm{B}$ cells were present. A higher proportion of the $T$ cells in the BAL of co-infected mice were $\mathrm{CD}^{+}$than $\mathrm{CD}^{+}$(Figure 5A). Therefore, we investigated the possibility that lethality was mediated by activity of cytotoxic T cells. FasL is one of the molecules that mediates apoptosis by cytotoxic $T$ cells and has been implicated in inducing the death of lung parenchymal cells in acute lung injury. ${ }^{45} \mathrm{~A}$ preliminary expression analysis showed that FasL was expressed in the lungs of coinfected mice (data not shown). Therefore, we infected mice lacking the Fas receptor (Fas-1-) sequentially with influenza and Hib and observed them for 25 days to determine whether the absence of FasL-mediated apoptosis would protect from lethality. As shown in Figure 6A, although resistant to single infections
A

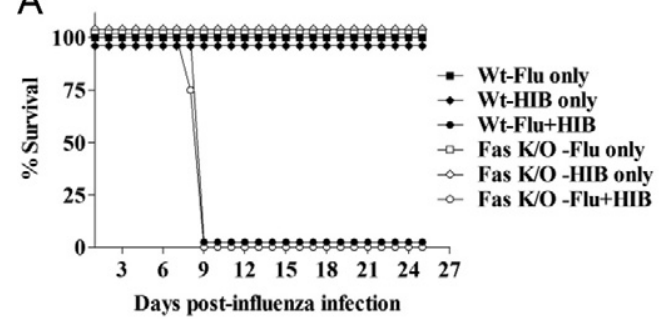

B
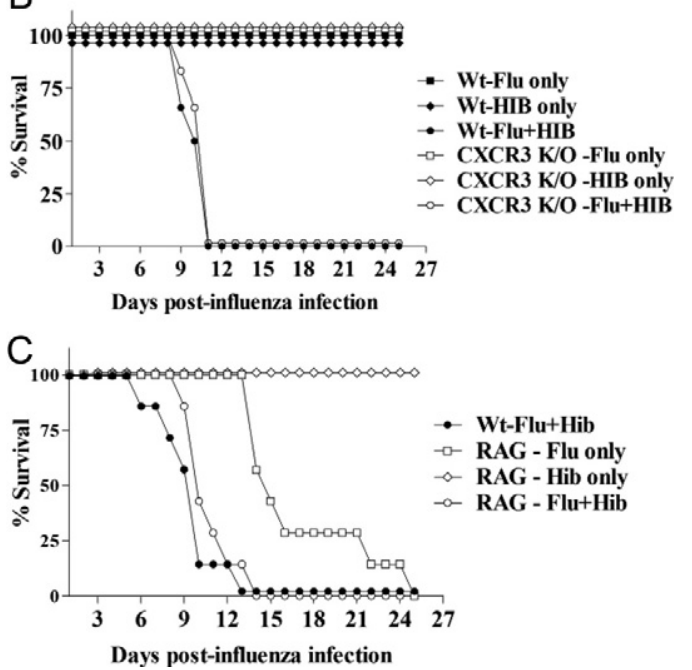

Figure 6. Lethal synergy between influenza virus and Hib is not dependent on Fas, CXCR3, and T and B cells. Groups of three or four knockout mice were infected with either Flu or Hib or sequentially infected with both agents 3 days apart. As controls, groups of three or four wild-type C57BL/ 6 mice were also infected with both agents 3 days apart, at the same doses used for the knockout mice (B6-Flu + Hib). The mice were monitored daily and the number of days survived after flu infection was recorded. The experiment was performed twice for each knockout strain and the figures show the percentage of total mice alive in each group at each time point postinfection ( $N=6-8$ mice per group in total). A: Survival of Fas $-/-$ mice after sequential infection with flu and Hib. Fas $-/-$ mice were infected i.n. with 30 PFU of Flu (Flu only) or $10^{5}$ CFU Hib (Hib only) or both agents (Flu + Hib) There was no significant difference in survival between wild-type and Fas $-/-$ mice in any of the groups. B: Survival of CXCR3 $-/-$ mice after sequential infection with flu and Hib. CXCR3 $-/-$ mice were infected i.n. with 30 PFU Flu (Flu only) or $10^{5}$ CFU Hib (Hib only) or both agents (Flu + Hib). There was no significant difference in survival between wild-type and CXCR3 $-/-$ mice in any of the groups. C: Survival of RAG $-/-$ mice after sequential infection with Flu and Hib. RAG $-/-$ mice were infected i.n. with 15 PFU Flu (Flu only) or $10^{5}$ CFU Hib (Hib only) or both agents (RAG-Flu + Hib). The number of days survived by $\mathrm{RAG}-/-$ mice infected with both agents and those infected with flu only was compared in a Mann-Whitney rank sum test and was significantly different $(P=0.0006)$. The number of days survived by double infected RAG-/- and wild-type mice was also compared by the same test and was not significantly different.

with low doses of influenza or Hib, mice that lacked Fas still succumbed to influenza-Hib co-infection, with kinetics that were similar to those observed in wild-type mice. This shows that damage to the lungs leading to lethality was not mediated through Fas-induced apoptosis.

ELISA data also indicated that the chemokine, IP-10 (CXCL10) was differentially expressed in doubly and singly infected mice (Figure 5B). IP-10, and related chemokines MIG (CXCL9) and I-TAC (CXCL11), bind to the CXCR3 chemokine receptor, which is involved in the recruitment of natural killer (NK) cells and activated $\mathrm{T}$ cells. To determine the role of CXCR3 and its chemokine ligands in the lethal synergy between influenza and Hib, 
CXCR3 - / - mice were infected with influenza and Hib as before. As shown in Figure 6B, the knockout mice did not succumb to infections with influenza or Hib only. However, sequential infection with both agents resulted in $100 \%$ lethality in CXCR3-/- mice, which also occurred with kinetics similar to those in wild-type mice.

As we had demonstrated that lethality was not induced by Fas-mediated apoptosis or CXCR3-mediated recruitment of $T$ cells at the site of infection, to further investigate the role of adaptive immunity in mediating the lethal synergy, RAG-/- mice, which lack $T$ and $B$ cells, were infected with influenza only or given both agents 3 days apart. A lower dose of influenza (15 PFU) was used, as influenza alone is lethal in these mice at higher doses, thus the use of a lower dose would prolong the survival of influenza-only mice, thereby enabling the distinction to be made between lethality mediated by influenza only or by sequential dual infection. The survival of the doubleinfected mice was monitored and compared with that of co-infected wild-type mice and RAG-/- mice that were only infected with influenza or Hib. RAG-/- mice appear to be resistant to infection with $10^{5} \mathrm{CFU}$ of $\mathrm{Hib}$ and did not exhibit any signs of illness (Figure $6 \mathrm{C}$ ). This surviving group of RAG - / - mice was sacrificed at the end of each experiment and the spleens were examined. The greatly reduced size of the spleen was consistent with the RAG-/- phenotype. As previously observed, RAG-/mice infected with influenza only were not able to control influenza and ultimately succumbed to the infection after 14-25 days. By contrast, the infection with two agents accelerated the time of death in RAG-/- mice to between 10 and 14 days. The difference was found to be statistically significant ( $P=0.0006$, Mann-Whitney rank sum test). However, there was no difference in the mortality and mean survival time of double-infected RAG-/and wild-type C57BL/6 mice. All of the mice in both groups died between 10-14 days postinfection with influenza, indicating that lethal synergy did not require the involvement of $\mathrm{T}$ or $\mathrm{B}$ cells.

\section{CCR2-Mediated Recruitment into the Lung Does Not Play an Essential Role in the Development of Lethal Synergy}

As our experiments indicate that the adaptive immune system does not play a role in mediating lethality in co-infected mice, we investigated the contribution of the early or innate immune system in causing lethality. Infection with influenza virus results in the up-regulation of a range of molecules in the lung, including the chemoattractant MCP-1, which serves to recruit monocytes and macrophages to the tissue via the presence of its receptor CCR2, which is expressed on these cells. ${ }^{46,47}$ Our data shows that MCP-1 is present in the BAL of both singly and doubly infected mice (Figure 5B). To explore the possibility that lethality may be due to increased trafficking of these activated cells into the lung, leading to excessive inflammation, mice deficient in the CCR2 receptor were infected with influenza virus or Hib only or with both agents sequentially. As shown in Figure 7, the

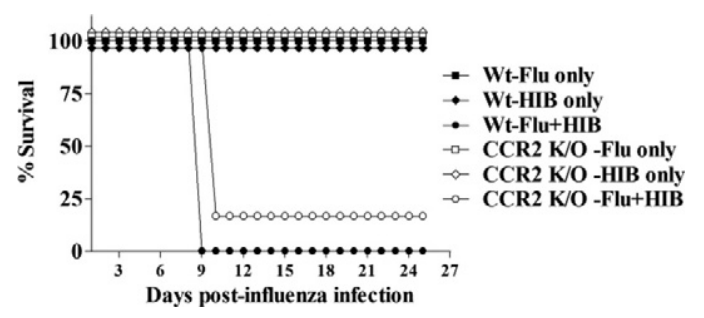

Figure 7. Lethal synergy between influenza virus and Hib is not dependent on CCR2. Groups of three or four CCR2 $-/-$ mice were infected i.n. with 30 PFU Flu (Flu only) or Hib (Hib only) or both agents 3 days apart (Flu + Hib). A group of three or four wild-type C57BL/ 6 mice was also infected with both agents 3 days apart as a control (B6-Flu + Hib). The mice were monitored daily and the number of days survived after flu infection was recorded. The figure shows the percentage of total mice alive in each group at each time point postinfection. Combined data from two independent experiments are shown. There was no significant difference in survival between wild-type and CCR2 $-/-$ mice in any of the groups.

lack of CCR2 did not protect the mice from lethality induced by sequential influenza virus and Hib infection. Again, there was no difference in the length of time that CCR2-/- and wild-type mice survived after dual infection, suggesting that recruitment of CCR2 positive cell subsets into the lungs of dual-infected mice does not play a role in causing lethality.

The Proinflammatory Cytokines TNF and IL-6 Are Not Essential for the Development of Lethal Synergy

Single infections with influenza virus or Hib induce production of the proinflammatory cytokine TNF. ${ }^{48-50}$ Under certain circumstances, excessive production of TNF induced by the synergistic effect of two separate agents results in immunopathology leading to death of the host, for example following the treatment of influenza-infected mice with staphylococcal enterotoxin B. ${ }^{51}$ TNF is also a major mediator of septic shock. We were able to detect this cytokine in BAL fluid from co-infected mice (Figure 5B). The role of TNF in lethal synergy between influenza and Hib was investigated in mice that lacked both TNF receptors 1 and 2 (TNFR1R2-I-), and were thus unable to respond to TNF. While these knockout mice were not affected by single infection with Hib, they were more sensitive than wild-type mice to influenza. Therefore, the dose of influenza virus used in this study was reduced to 20 PFU. This dose resulted in the death of approximately $25 \%$ of the TNFR1R2 $-/-$ mice in the absence of Hib co-infection. TNFR1R2-/- mice were sequentially infected with the two agents and their survival monitored for 25 days and compared with that of similarly infected wild-type mice and TNFR1R2-/- mice infected only with influenza virus or only with Hib. Control wild-type mice were also infected with the reduced dose (20 PFU) of influenza followed by Hib 3 days later. There was no difference in the mortality or mean survival times of double-infected TNFR1R2-I- and wild type mice (Figure $8 \mathrm{~A}$ ), suggesting that TNF production was not a contributing factor to the lethal synergistic effect.

The cytokine IL-6 is up-regulated in the lungs during both infection with influenza alone and co-infection with 
A

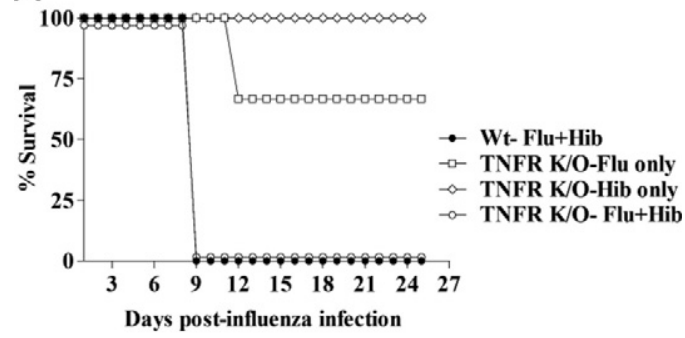

B

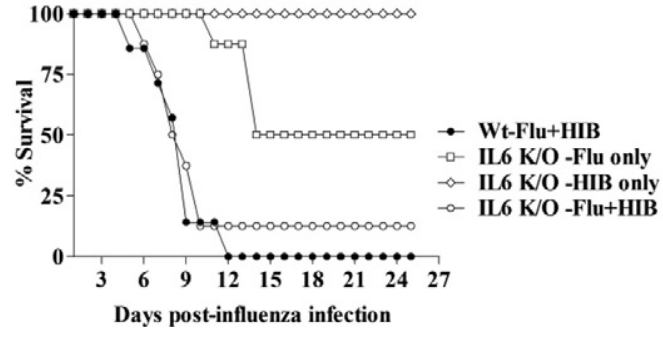

Figure 8. Lethal synergy between influenza virus and Hib is not dependent on TNF and IL-6. Groups of three or four mice lacking both TNF receptors (TNFR1R2-/-) or IL-6 (IL-6-/-) were infected i.n. with Flu or $10^{5}$ CFU Hib only or both agents 3 days apart. A group of three or four wild-type C57BL/ 6 mice was also infected with both agents 3 days apart as control (B6-Flu + $\mathrm{Hib})$. As the knockout mice were more susceptible to influenza, a lower dose of influenza was administered to all mice to minimize lethality resulting from influenza-only infection. The mice were monitored daily and the number of days survived after flu infection was recorded. The experiment was performed twice for each knockout strain. A: TNFR1R2-/- mice were infected i.n. with 20 PFU Flu (Flu only) or $10^{5}$ CFU Hib (Hib only) or both agents (Flu + Hib). Wild-type control mice were also infected with both agents at the same doses. A representative experiment is shown. There was a statistically significant difference in survival of the TNFR1R2 $-/-$ mice infected with flu alone and Flu $+\mathrm{Hib}(P<0.05$, rank sum test $)$. In contrast there was no significant difference in survival of TNFR1R2-/- and wild-type mice infected with both agents. B: Survival of IL6-/ - mice after sequential infection with flu and Hib. Groups of IL6-/- mice were infected i.n. with 15 PFU Flu (Flu only) or $10^{5}$ CFU Hib (Hib only) or both agents (Flu + Hib). Data combined from two independent experiments are shown. The number of days survived by IL-6-/- mice in the double-infected group was compared against the number of days survived by mice in the flu only group in a rank sum test and was significantly different $(P<0.01)$. The number of days survived by mice in the double-infected IL-6-/- group was also compared against double-infected wild-type C57BL/ 6 group by the same test and was not significantly different $(P=0.9)$.

Hib (Figure 8B). IL-6 is reported to possess both pro and anti-inflammatory properties and is responsible for inducing fever and the acute phase proteins in response to inflammation. ${ }^{52,53}$ Mice that lack IL-6 were found to be more susceptible to influenza and so the initial dose was reduced to $15 \mathrm{PFU}$ in this study, which was still lethal in approx $50 \%$ of mice infected with influenza virus only. Hib infection alone was not lethal to IL-6-/- mice. Even so, as shown in Figure 8B, IL-6-/- mice that were sequentially infected with influenza virus and Hib experienced a significantly accelerated demise compared with their influenza-only infected counterparts ( $P=0.002$, MannWhitney rank sum test). There was no difference in the lethality or mean survival time of double-infected IL-6-/and wild-type positive controls, indicating that IL-6 did not play an essential role in lethal synergy $(P=0.9)$.

\section{Lethality Is Not Mediated via Activation of TLR4}

Hib is a Gram-negative bacterium possessing a lipopolysaccharide (LPS)-containing cell membrane. It has been

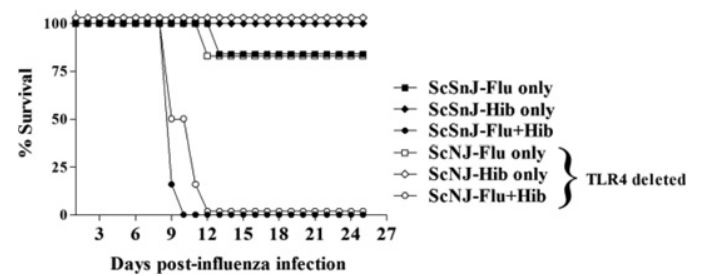

Figure 9. Lethal synergy between influenza and Hib does not require TLR4. Groups of three $\mathrm{C} 57 \mathrm{BL} / 10 \mathrm{ScNJ}$ (TLR4-/-) mice or the wild type strain C57BL/10ScSnJ were infected i.n. with 30 PFU Flu (Flu only) or Hib (Hib only) or both agents 3 days apart (Flu $+\mathrm{Hib})$. The mice were monitored daily and the number of days survived after flu infection was recorded. The figure shows the combined data from two separate experiments expressed as the percentage of total mice alive in each group at each time point postinfection $(N=6)$. There was no significant difference in survival between C57BL $10 \mathrm{ScNJ}$ and $\mathrm{C} 57 \mathrm{BL} / 10 \mathrm{ScSnJ}$ mice in any of the groups.

previously reported that infection with lymphocytic choriomeningitis virus, an RNA virus, rendered mice more sensitive to LPS-mediated toxic shock. ${ }^{54,55}$ TLR4-/mice are resistant to endotoxic shock. ${ }^{56,57}$ Therefore, to determine whether the lethal effects were mediated by the innate immune system activated by TLR4-dependent effects of LPS, mice deficient in the TLR4 receptor were infected with influenza and Hib (Figure 9). Surprisingly, there was no difference in the mortality and mean survival times of double-infected TLR4-/- mice and their wild type counterparts. Double-infected mice from both groups survived for 9-12 days postinfection, indicating that lethality occurs in a TLR4-independent manner.

\section{Discussion}

We have demonstrated that two common human respiratory pathogens, influenza and the bacterium $H$. influenzae, under certain circumstances of co-infection, are able to interact synergistically in the murine host to cause more severe disease, eventually leading to mortality. While $H$. influenzae b and influenza A/PR8 are not highly pathogenic in humans, we believe that this mouse model may reflect interactions in humans between more highly pathogenic strains of influenza and strains of $H$. influenzae to which there is no pre-existing immunity in the human host (generally the non-typable strains). In support of this idea, a study published during the 1918 influenza pandemic $^{38}$ demonstrated lethal synergism between viral and $H$. influenzae strains obtained from influenza victims, when these agents were administered to guinea pigs. While most strains of influenza virus predispose only vulnerable individuals to severe secondary bacterial infections, highly pathogenic pandemic strains may predispose even normal healthy adults to lethal superinfection. For example, in the1918 influenza pandemic, a high proportion of victims were previously healthy young adults who succumbed to secondary bacterial infections. ${ }^{58-62}$ Furthermore, historically, H. influenzae (also referred to as Pfeiffer's bacillus or Bacillus influenzae) was so often isolated from the sputum and autopsied lungs of patients during influenza outbreaks that it was initially proposed to be the causative agent of influenza. ${ }^{24}$ The high replicative capacity of pandemic influenza virus 
strains may also result in mortality in previously healthy adults in the absence of secondary bacterial infection, as we observe with high doses of influenza A/PR8 in mice. Presumed "sterile" deaths from influenza alone were documented during the 1918 influenza pandemic, although the majority of deaths appear to have resulted from secondary bacterial infections. ${ }^{16}$ Therefore, while infection with a high dose (or with a rapidly replicating strain) of influenza virus may cause lethality on its own, co-infection with $H$. influenzae significantly reduces the initial dose of influenza needed to cause lethality.

The pathological changes seen in this animal model of combined viral and bacterial pneumonia also evoke the features seen in the lungs of patients who died in the influenza pandemic of $1918-19 .{ }^{17,63}$ In particular, the prominent neutrophilic infiltrates in the bronchi and alveoli, severe denudation of the bronchial epithelium and confluent pneumonia observed in our co-infected mice recapitulate the histopathological findings in the Spanish flu victims. ${ }^{17,63}$ Although bronchial epithelial cell apoptosis appeared in both the combined and the influenza virus only mice, the process was delayed in the mice infected with influenza virus alone and was accompanied by signs of regeneration.

Examination of hematoxylin and eosin-stained sections of dual-infected lungs suggests that the pathological effects of dual infection occur early in infection, as bronchial necrosis, peribronchial inflammation and bronchitis was observed as early as day 4 postinfluenza infection or 1 day after Hib infection, and eventually led to epithelial erosion. In contrast, the lungs of singly infected mice displayed mild bronchiolitis at day 4 and were recovering by day 9. Thus, combination of both agents acts as a potent inducer of bronchial epithelial apoptosis, resulting in loss of the bronchial and bronchiolar epithelium. Without this natural barrier to infection, the bacteria are not expelled and are retained in the lungs; the mice were ultimately killed by confluent bronchopneumonia secondary to the infection. With respect to planning for an influenza pandemic, if the early lung damage seen in our mouse model is recapitulated in human patients, this would suggest that prophylactic treatment of individuals exposed to highly pathogenic influenza strains with antibiotics might be the most effective course. However, in more recent influenza pandemics, later antibiotic treatment has also been shown to be beneficial, but does not prevent all deaths.

Our experiments indicate that the interval between exposure to influenza virus and $H$. influenzae is a critical determinant of mortality. No synergistic effect was observed when both agents were administered at the same time, or when the interval was extended beyond 7 days. Peak lethality occurred when the agents were administered 3-4 days apart. The findings are in accordance with observations from an early study by Francis and de Torregrossa ${ }^{41}$ who reported that inoculating sublethal doses of influenza virus and clinical isolates of $H$. influenzae via the i.n. route either simultaneously or within 24 hours of each other did not cause death in animals but mortality occurred in the majority of the mice when the interval was increased to 2-5 days. In our studies, mortality increased as the interval was extended and peaked at an interval of 3-4 days, after which increasing the time interval between infections reduced the level of lethality. This suggests that the factor(s) involved in the lethal synergism have peak responses between 3 and 4 days after influenza infection. This tends to exclude the loss of cilia, which persists for 10-14 days after infection with influenza alone. ${ }^{64}$ One reason for the disease exacerbation may be the persistence of $H$. influenzae in the lung. Although double-infected mice were able to control the viral infection as well as mice infected with influenza only, they were not able to clear the bacterial infection as viable $H$. influenzae could be recovered from the lungs of the dying animals 6 days after infection. In contrast, no bacteria were recovered from the lungs of mice infected with $H$. influenzae only beyond 24 hours after infection, suggesting that a prior infection with influenza virus promotes the capacity of $H$. influenzae to persist in the lung and thereby increases the severity of the disease.

We investigated the interactions that resulted in lethality by using knockout mice. $H$. influenzae is a Gram-negative organism containing various cellular components such as LPS, which has been implicated in promoting inflammatory responses including the up-regulation of TNF via activation of TLR4 in septic shock. ${ }^{56,57,65,66}$ LPS has also been shown to have a potentiating effect on influenza induced TNF production by macrophages, ${ }^{67}$ and lymphocytic choriomeningitis virus has been shown to sensitize mice to low doses of LPS. ${ }^{68,69}$ This correlated with increased TNF production, which occurred by a T cell-dependent mechanism in some studies ${ }^{51,55,68}$ while others have suggested a $T$ cell-independent mechanism. ${ }^{54,69}$ We therefore explored the possibility that dual-infection was causing death through a mechanism involving TLR4 and TNF. However, mice that lacked the TLR4 receptor and were therefore protected from endotoxic shock still died after double infection, as did mice that lacked both TNF receptors and were thus insensitive to TNF. This indicated that lethal synergy in this model was not mediated by LPSinduced TNF production. Infection with influenza virus has been reported to induce a cytokine storm, which may lead to death via immunopathology. ${ }^{70-73}$ Along with TNF, the cytokine IL-6 have been implicated in this cytokine storm. ${ }^{73}$ However, using IL-6-/- mice, we found that deficiency in IL-6 production alone was not sufficient to ablate lethality after dual infection suggesting neither IL-6 nor TNF is essential for lethal synergy to occur.

Animal models have previously been used to investigate dual infections with influenza virus and respiratory bacteria $S$. pneumoniae, S. aureus, or S. pyogenes. ${ }^{9,10,15,21-23,74-76}$ A common feature is that, as in our model, infection with influenza appears to increase or prolong bacterial growth. Various explanations have been proposed for the predisposition to secondary bacterial infection induced by influenza infection in these models. These include effects on macrophages, such as decreased phagocytic activity ${ }^{21,22}$ and recruitment. ${ }^{77,78}$ In contrast, it has been reported that mice lacking the CCR2 chemokine receptor, a major chemotactic receptor for macrophages, are relatively more resistant to challenge with high doses of influenza virus as they are protected 
from influenza-induced immunopathology due to defective recruitment of $\mathrm{CCR}^{+}{ }^{+}$leukocytes into the lung. ${ }^{79,80}$ However, we found that CCR2-/- mice were not protected from the lethal effects of influenza virus and $\mathrm{Hib}$ dual infection, thereby ruling out an essential role for CCR2-induced leukocyte recruitment in mortality.

Recent studies have also suggested a T cell-mediated cytokine-dependent decrease in macrophage phagocytic activity during influenza virus and $S$. pneumoniae co-infection. ${ }^{81}$ Treatment of lymphocytic choriomeningitis virus-infected mice with staphylococcal enterotoxin B was also shown to increase cytokine production and to induce a fatal systemic shock-like syndrome, which could be partially inhibited by blocking TNF action. ${ }^{82}$ Similarly, staphylococcal enterotoxin B treatment synergized with influenza virus infection resulting in increased lung pathology and mortality. ${ }^{51}$ In both cases, staphylococcal enterotoxin B appeared to act in a T cell-dependent manner. However, in our current study, we found that RAG-/- mice lacking $T$ and $B$ cells were not protected from lethal synergy between influenza virus and $H$. influenzae and death occurred over an identical time course to that of double-infected wild-type controls. In contrast, RAG-/- mice infected with influenza virus alone died significantly later, while those infected with $\mathrm{Hib}$ alone survived. It therefore appears that synergistic lethality is mediated by an innate immune mechanism that is not absolutely dependent on TNF, IL-6, or TLR4. However, we cannot completely exclude the possibility that these molecules, while not essential, contribute to lethality in wild-type mice via redundant or overlapping pathways.

Virus-induced depression of neutrophil function or recruitment has also been suggested as a possible mechanism for increased bacterial growth and consequent pathogenic effects. Type I interferon, produced in response to lymphocytic choriomeningitis virus infection was shown to inhibit neutrophil recruitment from the bone marrow thus predisposing mice to Listeria monocytogenes infection. ${ }^{83}$ Another study suggested that sustained desensitization to bacterial TLR ligands after the resolution of influenza infection caused reduced neutrophil recruitment and increased bacterial load, but the effect of TLR densensitization or the increased bacterial load on survival was not tested directly. ${ }^{84}$ The effect on neutrophil recruitment lasted for at least 6 weeks, whereas in our model no lethality was seen when the bacteria were given 10 days or more after infection. Similarly, in humans, secondary bacterial infections normally occur within the first 2 weeks following influenza infection. ${ }^{59}$ As large numbers of neutrophils are recruited into the lung during influenza virus infection, ${ }^{85,86}$ an influenzamediated decrease in neutrophil recruitment seems an unlikely explanation for lethal synergy between influenza virus and $H$. influenzae. Furthermore, we, and others, ${ }^{9,10,23,74-76}$ have observed large numbers of infiltrating cells, including neutrophils in the lungs of mice during secondary bacterial infection. In addition, extensive neutrophilic infiltrates were prominent in postmortem findings for victims of the 1918 influenza pandemic. ${ }^{17}$ Based on these observations, it appears unlikely that decreased neutrophil recruitment to the lungs can explain our findings.

Further work is required to delineate the cellular and molecular mechanisms underlying lethal synergy between influenza virus and $H$. influenzae. Our work suggests a role for innate immunity and we will continue to investigate the role of various cells of the innate immune system including macrophages, neutrophils and NK cells. However, we have not completely excluded physical mechanisms that increase bacterial adherence to the lungs or that influence bacterial invasion in other ways. Evidence suggests that pneumococcal bacterial invasion is triggered by cleavage of protective sialic acid residues by the influenza neuraminidase enabling the bacteria to adhere to host cells. ${ }^{87,88} \mathrm{~A}$ role for influenza-induced cilia loss in the increased adhesion of $S$. pneumoniae to epithelial cells has also been documented. ${ }^{89,90}$ In contrast, Bakaletz et $\mathrm{al}^{40}$ found that influenza decreased adherence of $H$. influenzae to respiratory epithelium and that the bacteria preferentially bound to ciliated cells. Furthermore, neuraminidase treatment of epithelial cells has been reported to decrease the adhesion of $\mathrm{H}$. influenzae. ${ }^{91}$ However, roles for innate immunity and physical mechanisms are not mutually exclusive. For example, innate immune cells might damage the epithelium in a manner that facilitates bacterial invasion.

In conclusion, we have developed a robust model of lethal synergy between influenza virus and the bacterial respiratory pathogen, $H$. influenzae that is mediated by innate immunity, but does not require the TLR4-dependent pathway of TNF induction. This model will be useful for further investigating the interaction between these agents, which may have resulted in numerous deaths during the 1918 influenza pandemic and continues to constitute a significant clinical problem in susceptible individuals. ${ }^{5,92,93}$

\section{References}

1. Simonsen L: The global impact of influenza on morbidity and mortality. Vaccine 1999, 17(Suppl 1):S3-S10

2. Thompson WW, Shay DK, Weintraub E, Brammer L, Bridges CB: Cox NJ, Fukuda K: Influenza-associated hospitalizations in the United States. JAMA 2004, 292:1333-1340

3. Wong CM, Chan KP, Hedley AJ, Peiris JS: Influenza-associated mortality in Hong Kong. Clin Infect Dis 2004, 39:1611-1617

4. Yap FH, Ho PL, Lam KF, Chan PK, Cheng YH, Peiris JS: Excess hospital admissions for pneumonia, chronic obstructive pulmonary disease, and heart failure during influenza seasons in Hong Kong. J Med Virol 2004, 73:617-623

5. Sethi S: Bacterial pneumonia. Managing a deadly complication of influenza in older adults with comorbid disease Geriatrics 2002, 57:56-61

6. Hament JM, Kimpen JL, Fleer A, Wolfs TF: Respiratory viral infection predisposing for bacterial disease: a concise review. FEMS Immunol Med Microbiol 1999, 26:189-195

7. Loosli CG: Influenza and the interaction of viruses and bacteria in respiratory infections. Medicine (Baltimore) 1973, 52:369-384

8. Seki M, Kosai K, Yanagihara K, Higashiyama Y, Kurihara S, Izumikawa K, Miyazaki Y, Hirakata Y, Tashiro T, Kohno S: Disease severity in patients with simultaneous influenza and bacterial pneumonia. Intern Med 2007, 46:953-958

9. McCullers JA, Webster R: A mouse model of dual infection with influenza virus and Streptococcus pneumoniae. Options for control of 
influenza IV. Edited by Osterhaus A, Cox N, Hampson A. Amsterdam. Elsevier Science, 2001, pp 601-607

10. Okamoto S, Kawabata S, Nakagawa I, Okuno Y, Goto T, Sano K, Hamada S: Influenza A virus-infected hosts boost an invasive type of Streptococcus pyogenes infection in mice. J Virol 2003, 77: 4104-4112

11. Alonso JM, Guiyoule A, Zarantonelli ML, Ramisse F, Pires R, Antignac A, Deghmane AE, Huerre M, van der Werf S, Taha MK: A model of meningococcal bacteremia after respiratory superinfection in influenza A virus-infected mice. FEMS Microbiol Lett 2003, 222:99-106

12. Jarstrand $C$, Tunevall $G$ : The influence of bacterial superinfection on the clinical course of influenza. Studies from the influenza epidemics in Stockholm during the winters $1969-70$ and 1971-72. Scand J Infect Dis 1975, 7:243-247

13. Schwarzmann SW, Adler JL, Sullivan RJ Jr, Marine WM: Bacterial pneumonia during the Hong Kong influenza epidemic of 1968-1969. Arch Intern Med 1971, 127:1037-1041

14. Kilbourne ED: Influenza. New York, Plenum, 1987, pp 220-240

15. McCullers JA: Insights into the interaction between influenza virus and pneumococcus. Clin Microbiol Rev 2006, 19:571-582

16. Morens DM, Taubenberger JK, Fauci AS: Predominant role of bacterial pneumonia as a cause of death in pandemic influenza: implications for pandemic influenza preparedness. J Infect Dis 2008, 198:962-970

17. Taubenberger JK, Morens DM: The pathology of influenza virus infections. Annu Rev Pathol 2008, 3:499-522

18. Brundage JF, Shanks GD: Deaths from bacterial pneumonia during 1918-19 influenza pandemic. Emerg Infect Dis 2008, 14:1193-1199

19. Brundage JF, Shanks GD: What really happened during the 1918 influenza pandemic? The importance of bacterial secondary infections. J Infect Dis 2007, 196:1717-1719

20. Levine J, Buchman CA, Fregien N: Influenza A virus infection of human Schwann cells in vitro. Acta Otolaryngol 2003, 123:41-45

21. Wyde PR, Six HR, Ambrose MW, Throop BJ: Influenza virus infection and bacterial clearance in young adult and aged mice. J Gerontol 1989, 44:B118-B124

22. Nickerson CL, Jakab GJ: Pulmonary antibacterial defenses during mild and severe influenza virus infection. Infect Immun 1990, $58: 2809-2814$

23. Gardner ID, Kung TM: Histopathological changes in the lungs of influenza-infected mice superinfected with Staphylococcus aureus. Br J Exp Pathol 1980, 61:415-420

24. Aubrey R, Tang C: The pathogenesis of disease due to Type b Haemophilus influenzae. Methods in Molecular Medicine, vol 71: Haemophilus influenzae Protocols. Edited by Herbert M, Hood DW, Moxon ER. Totowa, NJ, Humana Press, 2002, pp 29-50

25. Chin CL, Manzel LJ, Lehman EE, Humlicek AL, Shi L, Starner TD, Denning GM, Murphy TF, Sethi S, Look DC: Haemophilus influenzae from patients with chronic obstructive pulmonary disease exacerbation induce more inflammation than colonizers. Am J Respir Crit Care Med 2005, 172:85-91

26. Masuda K, Masuda R, Nishi J, Tokuda K, Yoshinaga M, Miyata K: Incidences of nasopharyngeal colonization of respiratory bacterial pathogens in Japanese children attending day-care centers. Pediatr Int 2002, 44:376-380

27. Wilkinson TM, Hurst JR, Perera WR, Wilks M, Donaldson GC, Wedzicha JA: Effect of interactions between lower airway bacterial and rhinoviral infection in exacerbations of COPD. Chest 2006, 129:317-324

28. Grant GB, Campbell H, Dowell SF, Graham SM, Klugman KP, Mulholland EK, Steinhoff M, Weber MW, Qazi S: Recommendations for treatment of childhood non-severe pneumonia. Lancet Infect Dis 2009, 9:185-196

29. Murphy TF: Respiratory infections caused by non-typeable Haemophilus influenzae. Curr Opin Infect Dis 2003, 16:129-134

30. Rosenstein NE, Perkins BA: Update on Haemophilus influenzae serotype $b$ and meningococcal vaccines. Pediatr Clin North Am 2000, 47:337-352, vi

31. Burns IT, Zimmerman RK: Haemophilus influenzae type B disease, vaccines, and care of exposed individuals. J Fam Pract 2000, 49:S7-S14

32. Peltola $\mathrm{H}$ : Worldwide Haemophilus influenzae type $\mathrm{b}$ disease at the beginning of the 21st century: global analysis of the disease burden
25 years after the use of the polysaccharide vaccine and a decade after the advent of conjugates. Clin Microbiol Rev 2000, 13:302-317

33. Peltola VT, McCullers JA: Respiratory viruses predisposing to bacterial infections: role of neuraminidase. Pediatr Infect Dis J 2004, 23:S87-S97

34. Broker M: Burden of invasive disease caused by Haemophilus influenzae type b in Asia. Jpn J Infect Dis 2009, 62:87-92

35. Ulanova M, Tsang RS: Invasive Haemophilus influenzae disease: changing epidemiology and host-parasite interactions in the 21st century. Infect Genet Evol 2009, 9:594-605

36. Takano M, Ozaki K, Nitahara Y, Higuchi W, Takano T, Nishiyama A, Yamamoto T: Streptococcus pneumoniae and Haemophilus influenzae at the initial stage of influenza. Pediatr Int 2009, 51:687-695

37. Shope R: Swine Influenza III. Filtration experiments and etiology. J Exp Med 1931, 54:373-385

38. Orticoni M, Barbie M, Auge M: Pathogenie de la Grippe. La Presse Medical 1919, 26:247-249

39. Michaels RH, Myerowitz RL, Klaw R: Potentiation of experimental meningitis due to Haemophilus influenzae by influenza A virus. $J$ Infect Dis 1977, 135:641-645

40. Bakaletz LO, Hoepf TM, DeMaria TF, Lim DJ: The effect of antecedent influenza A virus infection on the adherence of Hemophilus influenzae to chinchilla tracheal epithelium. Am J Otolaryngol 1988, 9:127-134

41. Francis $T$, de Torregrosa MV: Combined infection of mice with $\mathrm{H}$. influenzae and influenza virus by the intranasal route. $\mathrm{J}$ Infect Dis 1944, 76:70-77

42. Tannock GA, Paul JA, Barry RD: Relative immunogenicity of the cold-adapted influenza virus A/Ann Arbor/6/60 (A/AA/6/60-ca), recombinants of $A / A A / 6 / 60-c a$, and parental strains with similar surface antigens. Infect Immun 1984, 43:457-462

43. Lysenko E, Richards JC, Cox AD, Stewart A, Martin A, Kapoor M, Weiser JN: The position of phosphorylcholine on the lipopolysaccharide of Haemophilus influenzae affects binding and sensitivity to C-reactive protein-mediated killing. Mol Microbiol 2000, 35:234-245

44. Sarawar SR, Doherty PC: Concurrent production of interleukin-2, interleukin-10, and gamma interferon in the regional lymph nodes of mice with influenza pneumonia. J Virol 1994, 68:3112-3119

45. Matute-Bello G, Liles WC, Steinberg KP, Kiener PA, Mongovin S, Chi $E Y$, Jonas M, Martin TR: Soluble Fas ligand induces epithelial cell apoptosis in humans with acute lung injury (ARDS). J Immunol 1999, 163:2217-2225

46. Wareing MD, Lyon AB, Lu B, Gerard C, Sarawar SR: Chemokine expression during the development and resolution of a pulmonary leukocyte response to influenza A virus infection in mice. J Leukoc Biol 2004, 76:886-895

47. Wareing MD, Lyon A, Inglis C, Giannoni F, Charo I, Sarawar SR: Chemokine regulation of the inflammatory response to a low-dose influenza infection in CCR2-/- mice. J Leukoc Biol 2007, 81:793-801

48. Kaufmann A, Salentin R, Meyer RG, Bussfeld D, Pauligk C, Fesq H, Hofmann P, Nain M, Gemsa D, Sprenger H: Defense against influenza A virus infection: essential role of the chemokine system. Immunobiology 2001, 204:603-613

49. Wang X, Moser C, Louboutin JP, Lysenko ES, Weiner DJ, Weiser JN, Wilson JM: Toll-like receptor 4 mediates innate immune responses to Haemophilus influenzae infection in mouse lung. J Immunol 2002, 168:810-815

50. Wieland CW, Florquin S, Maris NA, Hoebe K, Beutler B, Takeda K Akira S, van der Poll T: The MyD88-dependent, but not the MyD88independent, pathway of TLR4 signaling is important in clearing nontypeable haemophilus influenzae from the mouse lung. J Immunol 2005, 175:6042-6049

51. Zhang WJ, Sarawar S, Nguyen P, Daly K, Rehg JE, Doherty PC, Woodland DL, Blackman MA: Lethal synergism between influenza infection and staphylococcal enterotoxin B in mice. J Immunol 1996, 157:5049-5060

52. Conn CA, McClellan JL, Maassab HF, Smitka CW, Majde JA, Kluger $\mathrm{MJ}$ : Cytokines and the acute phase response to influenza virus in mice. Am J Physiol 1995, 268:R78-R84

53. Kaiser L, Fritz RS, Straus SE, Gubareva L, Hayden FG: Symptom pathogenesis during acute influenza: interleukin- 6 and other cytokine responses. J Med Virol 2001, 64:262-268

54. Doughty L, Nguyen K, Durbin J, Biron C: A role for IFN-alpha beta in virus infection-induced sensitization to endotoxin. J Immunol 2001, 166:2658-2664 
55. Nguyen KB, Biron CA: Synergism for cytokine-mediated disease during concurrent endotoxin and viral challenges: roles for NK and T cell IFN-gamma production. J Immunol 1999, 162:5238-5246

56. Poltorak A, He X, Smirnova I, Liu MY, Van Huffel C, Du X, Birdwell D, Alejos E, Silva M, Galanos C, Freudenberg M, Ricciardi-Castagnoli P, Layton B, Beutler B: Defective LPS signaling in C3H/HeJ and C57BL/ 10ScCr mice: mutations in TIr4 gene. Science 1998, 282:2085-2088

57. Hoshino K, Takeuchi O, Kawai T, Sanjo H, Ogawa T, Takeda Y, Takeda K, Akira S: Cutting edge: toll-like receptor 4 (TLR4)-deficient mice are hyporesponsive to lipopolysaccharide: evidence for TLR4 as the Lps gene product. J Immunol 1999, 162:3749-3752

58. Brundage JF: Interactions between influenza and bacterial respiratory pathogens: implications for pandemic preparedness. Lancet Infect Dis 2006, 6:303-312

59. Brundage JF: Cases and deaths during influenza pandemics in the United States. Am J Prev Med 2006, 31:252-256

60. Kawana A, Naka G, Fujikura Y, Kato Y, Mizuno Y, Kondo T, Kudo K: Spanish influenza in Japanese armed forces, 1918-1920. Emerg Infect Dis 2007, 13:590-593

61. Taubenberger JK, Morens DM: 1918 Influenza: the mother of all pandemics. Emerg Infect Dis 2006, 12:15-22

62. Luk J, Gross P, Thompson WW: Observations on mortality during the 1918 influenza pandemic. Clin Infect Dis 2001, 33:1375-1378

63. Morens DM, Fauci AS: The 1918 influenza pandemic: insights for the 21st century. J Infect Dis 2007, 195:1018-1028

64. Harford CG aH, A: Effect of influenza virus on cilia and epithelial cells in the bronchi of mice. J Exp Med 1952, 95:173-190

65. Qureshi ST, Lariviere L, Leveque G, Clermont S, Moore KJ, Gros P, Malo D: Endotoxin-tolerant mice have mutations in Toll-like receptor 4 (TIr4). J Exp Med 1999, 189:615-625

66. Beutler B, Du X, Poltorak A: Identification of Toll-like receptor 4 (TIr4) as the sole conduit for LPS signal transduction: genetic and evolutionary studies. J Endotoxin Res 2001, 7:277-280

67. Nain M, Hinder F, Gong JH, Schmidt A, Bender A, Sprenger H, Gemsa D: Tumor necrosis factor-alpha production of influenza A virus-infected macrophages and potentiating effect of lipopolysaccharides. J Immunol 1990, 145:1921-1928

68. Nansen A, Christensen JP, Marker O, Thomsen AR: Sensitization to lipopolysaccharide in mice with asymptomatic viral infection: role of T cell-dependent production of interferon-gamma. J Infect Dis 1997, 176:151-157

69. Nansen A, Randrup Thomsen A: Viral infection causes rapid sensitization to lipopolysaccharide: central role of IFN-alpha beta. J Immunol 2001, 166:982-988

70. Cheung CY, Poon LL, Lau AS, Luk W, Lau YL, Shortridge KF, Gordon $S$, Guan Y, Peiris JS: Induction of proinflammatory cytokines in human macrophages by influenza A (H5N1) viruses: a mechanism for the unusual severity of human disease? Lancet 2002, 360:1831-1837

71. Chan MC, Cheung CY, Chui WH, Tsao SW, Nicholls JM, Chan YO, Chan RW, Long HT, Poon LL, Guan Y, Peiris JS: Proinflammatory cytokine responses induced by influenza A (H5N1) viruses in primary human alveolar and bronchial epithelial cells. Respir Res 2005, 6:135

72. Kash JC, Tumpey TM, Proll SC, Carter V, Perwitasari O, Thomas MJ, Basler CF, Palese P, Taubenberger JK, Garcia-Sastre A, Swayne DE, Katze MG: Genomic analysis of increased host immune and cell death responses induced by 1918 influenza virus. Nature 2006, 443:578-581

73. Kobasa D, Jones SM, Shinya K, Kash JC, Copps J, Ebihara H, Hatta $\mathrm{Y}, \mathrm{Kim} \mathrm{JH}$, Halfmann P, Hatta M, Feldmann F, Alimonti JB, Fernando L, Li Y, Katze MG, Feldmann H, Kawaoka Y: Aberrant innate immune response in lethal infection of macaques with the 1918 influenza virus. Nature 2007, 445:319-323

74. McCullers JA, Rehg JE: Lethal synergism between influenza virus and Streptococcus pneumoniae: characterization of a mouse model and the role of platelet-activating factor receptor. $\mathrm{J}$ Infect Dis 2002 186:341-350
75. LeVine AM, Koeningsknecht V, Stark JM: Decreased pulmonary clearance of S. pneumoniae following influenza A infection in mice. J Virol Methods 2001, 94:173-186

76. Speshock JL, Doyon-Reale N, Rabah R, Neely MN, Roberts PC: Filamentous influenza $A$ virus infection predisposes mice to fatal septicemia following superinfection with Streptococcus pneumoniae serotype 3. Infect Immun 2007, 75:3102-3111

77. Kleinerman ES, Snyderman R, Daniels CA: Depressed monocyte chemotaxis during acute influenza infection. Lancet 1975, 2:10631066

78. Kleinerman ES, Daniels CA, Polisson RP, Snyderman R: Effect of virus infection on the inflammatory response. Depression of macrophage accumulation in influenza-infected mice Am J Pathol 1976, 85: 373-382

79. Dawson TC, Beck MA, Kuziel WA, Henderson F, Maeda N: Contrasting effects of CCR5 and CCR2 deficiency in the pulmonary inflammatory response to influenza A virus. Am J Pathol 2000, 156: 1951-1959

80. Lin KL, Suzuki Y, Nakano H, Ramsburg E, Gunn MD: CCR2+ monocyte-derived dendritic cells and exudate macrophages produce influenza-induced pulmonary immune pathology and mortality. J Immunol 2008, 180:2562-2572

81. Sun K, Metzger DW: Inhibition of pulmonary antibacterial defense by interferon-gamma during recovery from influenza infection. Nat Med 2008, 14:558-564

82. Sarawar SR, Blackman MA, Doherty PC: Superantigen shock in mice with an inapparent viral infection. J Infect Dis 1994, 170:1189-1194

83. Navarini AA, Recher M, Lang KS, Georgiev P, Meury S, Bergthaler A, Flatz L, Bille J, Landmann R, Odermatt B, Hengartner $\mathrm{H}$, Zinkernagel RM: Increased susceptibility to bacterial superinfection as a consequence of innate antiviral responses. Proc Natl Acad Sci USA 2006, 103:15535-15539

84. Didierlaurent A, Goulding J, Patel S, Snelgrove R, Low L, Bebien M, Lawrence T, van Rijt LS, Lambrecht BN, Sirard JC, Hussell T: Sustained desensitization to bacterial Toll-like receptor ligands after resolution of respiratory influenza infection. J Exp Med 2008, 205: 323-329

85. Renegar KB: Influenza virus infections and immunity: a review of human and animal models. Lab Anim Sci 1992, 42:222-232

86. Wareing MD, Shea AL, Inglis CA, Dias PB, Sarawar SR: CXCR2 is required for neutrophil recruitment to the lung during influenza virus infection, but is not essential for viral clearance. Viral Immunol 2007, 20:369-378

87. McCullers JA, Bartmess KC: Role of neuraminidase in lethal synergism between influenza virus and Streptococcus pneumoniae. $J$ Infect Dis 2003, 187:1000-1009

88. Peltola VT, Murti KG, McCullers JA: Influenza virus neuraminidase contributes to secondary bacterial pneumonia. J Infect Dis 2005 192:249-257

89. Nugent KM, Pesanti EL: Tracheal function during influenza infections. Infect Immun 1983, 42:1102-1108

90. Plotkowski MC, Puchelle E, Beck G, Jacquot J, Hannoun C: Adherence of type I Streptococcus pneumoniae to tracheal epithelium of mice infected with influenza A/PR8 virus. Am Rev Respir Dis 1986, 134:1040-1044

91. Kawakami K, Ahmed K, Utsunomiya Y, Rikitomi N, Hori A, Oishi K, Nagatake T: Attachment of nontypable Haemophilus influenzae to human pharyngeal epithelial cells mediated by a ganglioside receptor. Microbiol Immunol 1998, 42:697-702

92. Memoli MJ, Morens DM, Taubenberger JK: Pandemic and seasonal influenza: therapeutic challenges. Drug Discov Today 2008, 13: 590-595

93. Active Bacterial Core Surveillance Report EIPN: Haemophilus influenzae 2006. Centers for Disease Control and Prevention, 2007, p 1 